\title{
Langasite Surface Acoustic Wave Gas Sensors: Modeling and Verification
}

\author{
P. Zheng,,$^{1,2 *}$ D.W. Greve, ${ }^{1,3}$ I.J. Oppenheim, ${ }^{1,4}$ \\ ${ }^{1}$ National Energy Technology Laboratory, Pittsburgh, PA, USA \\ ${ }^{2}$ Department of Physics, Carnegie Mellon University, Pittsburgh, PA, USA \\ ${ }^{3}$ Department of Electrical and Computer Engineering, Carnegie Mellon University, Pittsburgh, \\ PA, USA \\ ${ }^{4}$ Department of Civil and Environmental Engineering, Carnegie Mellon University, Pittsburgh, \\ PA, USA
}

\begin{abstract}
We report finite element simulations of the effect of conductive sensing layers on the surface wave velocity of langasite substrates. The simulations include both the mechanical and electrical influences of the conducting sensing layer. We show that three-dimensional simulations are necessary because of the out-of-plane displacements of the commonly used $(0,138.5,26.7)$ Euler angle. Measurements of the transducer input admittance in reflective delay-line devices yield a value for the electromechanical coupling coefficient that is in good agreement with the threedimensional simulations on bare langasite substrate. The input admittance measurements also show evidence of excitation of an additional wave mode and excess loss due to the finger resistance. The results of these simulations and measurements will be useful in the design of surface acoustic wave gas sensors.
\end{abstract}

* Present address: Covidien plc, Mail Stop A24, 5920 Longbow Drive, Boulder, CO 80301. 


\section{Introduction}

Wireless, harsh-environment sensing has attracted recent research interest because of applications in engines and combustion systems. Wireless sensing is advantageous when penetrations into the harsh environment are undesirable. Surface acoustic wave (SAW) sensors fabricated on high-temperature piezoelectric substrates represent an attractive solution because they combine sensing with a transponder function. Langasite SAW sensors have received particular attention recently for temperature [1,2,3,4,5] and gas [6,7,8,9] sensing. Elsewhere [10] we reported on experimental characterization of langasite surface acoustic wave (SAW) sensors for temperature and oxygen concentration. In this paper we will report on simulations of the performance of these sensors. We consider the effect of resistive overlayers on the surface acoustic wave velocity. Calculations are performed using finite element analysis which allows us to include the electrostatic and mechanical effects of the resistive overlayer and also a dielectric buffer layer. For the $(0,138.5,26.7)$ Euler angle it is necessary to perform a 3D simulation because of the non-zero out-of-plane displacements of the surface wave.

Another important practical issue is the input admittance of the SAW sensor. We will also report on measurements of the input admittance of several interdigitated transducer (IDT) designs. We will also show that the measured input admittance exhibits additional losses that are due to extrinsic losses in the fingers and connections.

\section{SAW Sensor Modeling}

We consider here a SAW device with a gas sensing layer $[10,11,12]$. SAW gas sensing can be accomplished using either gas- induced conductivity [11] or mass changes to the sensing layer covering the acoustic path [12]. Changes in the electrical conductivity or the mass density change the velocity of the surface acoustic wave, which can be detected in transient or steadystate measurements. Here we consider a metal oxide gas sensing layer. Metal oxides have been widely used in gas sensing over a wide range of temperatures and are particularly suitable for high-temperature sensing because many oxides are stable at elevated temperatures. Many metal oxides have an electrical conductivity that is strongly influenced by the oxygen vacancy 
concentration. In these oxides the steady state oxygen vacancy concentration is in equilibrium with the gas ambient, due to the reversible reaction

$$
O_{o}^{x} \leftrightarrow V_{\ddot{o}}+2 e^{\prime}+\frac{1}{2} O_{2}^{(g)}
$$

where $O_{o}^{x}$ is an oxygen atom on an oxygen site, $V_{\ddot{o}}$ is a doubly-positive oxygen vacancy, and $e^{\prime}$ is a negatively charged free electron. In an $n$-type oxide, it can be shown [13] that the electrical conductivity is given by

$$
\sigma=A e^{-E_{A} / k T} P_{o_{2}}^{1 / m}
$$

where $E_{A}$ is the activation energy for electrical conductivity, $P_{o_{2}}$ is the oxygen partial pressure, and $m$ is a constant typically between 4 and 6 .

When an oxygen defect conductor is used as the sensing layer the change in mass is very small. The electrical conductivity of the sensing layer influences the surface wave velocity through the electro-acoustic effect. Using perturbation theory it can be shown [11,14] that the change in surface wave velocity $\Delta v$ is given by

$$
\frac{\Delta v}{v_{0}}=\frac{-K^{2}}{2} \frac{1}{1+\left(\frac{v_{0} \varepsilon_{p}\left(k_{0}\right)}{\sigma_{0} t}\right)^{2}}
$$

where $K^{2}$ is the electromechanical coupling coefficient, $v_{0}$ is the surface acoustic wave velocity for a free surface, $\varepsilon_{p}\left(k_{0}\right)$ is the effective permittivity in the absence of the piezoelectric effect, $k_{0}$ is the wavenumber of the surface acoustic wave, $\sigma_{0}$ is the bulk conductivity of the sensing layer, and $t$ is the sensing layer thickness. While (3) provides an indication of the velocity change to be expected for a particular sensing layer, it does not include the effect of insulating spacer layers (which may be desirable to limit reactions between the ambient and the substrate) or the mechanical effect of the overlayers. Accordingly, we performed finite element simulations in 
order to take these phenomena into account. These calculations also yield insight into the generation of other wave types.

Finite element simulations were performed using COMSOL 3.5a in either the 2D piezoelectric plane strain (smppn) or 3D piezoelectric solid (smpz3d) modes. Material constants for langasite have been reported by Kosinski et al. [15] and Chilla et al. [16]. We used material constants from Chilla et al. [16] after transformation to the appropriate crystal axes and verified that the simulated surface wave velocities were in agreement with values in that paper. In this section we are concerned with determination of the surface acoustic wave velocity along various directions and with different surface layers. The surface wave velocity was determined by performing an eigenmode calculation for a small 2D or 3D section with periodic boundary conditions along the direction of propagation. As the 2D calculations were performed in the plane strain mode no displacement is allowed transverse to the direction of propagation. For the 3D simulations periodic boundary conditions were also imposed transverse to the direction of propagation. The eigenmodes corresponding to surface acoustic waves were then identified and the surface acoustic wave velocity $v=\lambda f$ calculated, where $\lambda$ is the periodic distance of the simulation model along the direction of propagation, and $f$ is the eigenfrequency result from simulation.

Figure 1 shows an eigenmode resulting from a 3D simulation corresponding to a surface acoustic wave for the $(0,138.5,26.7)$ direction in langasite with a free surface boundary condition. The color corresponds to the displacement amplitude normal to the surface and the distorted shape is proportional to the actual displacements. The displacements do not correspond to the classical Rayleigh wave because of the anisotropic character of the langasite crystal. In particular there is a nonzero displacement transverse to the direction of motion (apparent from the distorted shape in the top view). 

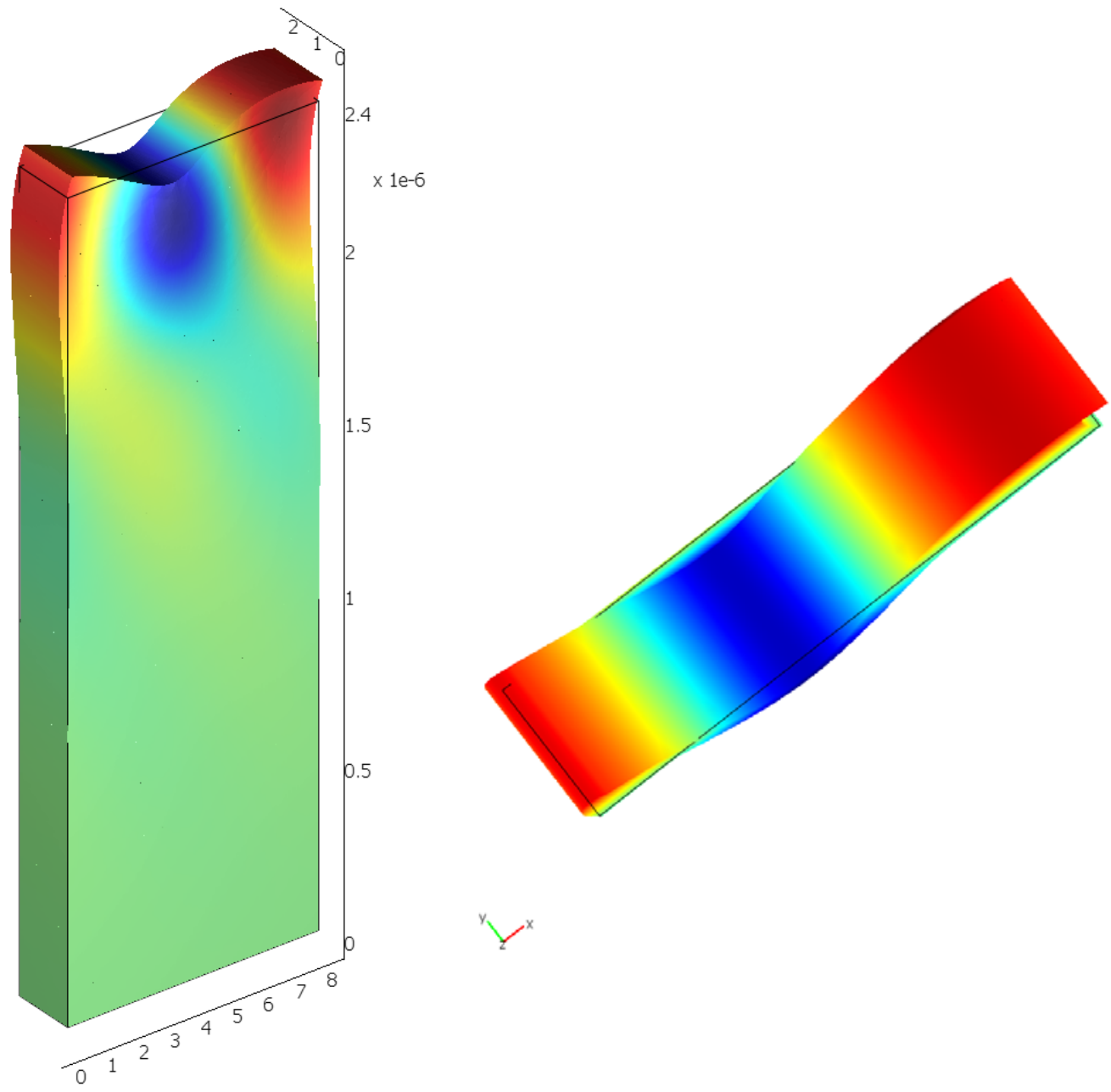

Figure 1. Eigenmode corresponding to a surface acoustic wave for the $(0,138.5,26.7)$ direction in langasite (zero surface charge; color indicates displacement normal to surface). The detail is the view normal to the top surface and shows a small displacement transverse to the direction of wave motion.

Results of these calculations for propagation along several Euler angles are shown in Table I. Also shown are published values for the SAW propagation velocities. It is apparent that in all cases better agreement is obtained using the $3 \mathrm{D}$ calculation. The $2 \mathrm{D}$ calculation is least accurate for propagation directions characterized by large $z$ displacement. 
TABLE I. PROPAGATION VELOCITY AND ELECTROMECHANICAL COUPLING COEFFICIENT ALONG DIFFERENT DIRECTIONS.

\begin{tabular}{|c|c|c|c|c|c|c|}
\hline Euler angle & $\begin{array}{l}\text { reported } \\
\text { velocity } \\
(\mathrm{m} / \mathrm{sec})\end{array}$ & $\begin{array}{l}\text { velocity } \\
2 \mathrm{D} \\
\text { simulation } \\
\text { (m/sec) }\end{array}$ & $\begin{array}{l}\text { velocity } \\
3 D \\
\text { simulation } \\
\text { (m/sec) }\end{array}$ & $\begin{array}{c}\text { reported } \\
\mathrm{K}^{2}(\%)\end{array}$ & $\begin{array}{c}\mathrm{K}^{2}(\%) \\
2 \mathrm{D} \\
\text { simulation }\end{array}$ & $\begin{array}{c}\mathrm{K}^{2}(\%) \\
3 \mathrm{D} \\
\text { simulation }\end{array}$ \\
\hline$(90,90,0)$ & $\begin{array}{c}2450[17] \\
{[16]}\end{array}$ & $\begin{array}{c}2553 \\
(+4 \%)\end{array}$ & $\begin{array}{c}2459 \\
(+0.4 \%)\end{array}$ & $0[17]$ & 0.0003 & 0.0038 \\
\hline$(90,90,90)$ & 2900 [17] & $\begin{array}{c}2975 \\
(+3 \%)\end{array}$ & $\begin{array}{c}2915 \\
+(0.5 \%)\end{array}$ & $\begin{array}{c}0.44[17] \\
0.35[18]\end{array}$ & 0.36 & 0.49 \\
\hline$(0,90,0)$ & 2320 [17] & $\begin{array}{c}2541 \\
(+10 \%)\end{array}$ & $\begin{array}{l}2350 \\
(+1 \%)\end{array}$ & $\begin{array}{c}0.38[17] \\
0.33[11] \\
{[18]}\end{array}$ & 0.35 & 0.42 \\
\hline$(0,0,0)$ & 2380 [17] & $\begin{array}{c}2956 \\
(+24 \%)\end{array}$ & $\begin{array}{c}2404 \\
(+1 \%)\end{array}$ & $0[17]$ & 0.01 & 0.001 \\
\hline$(0,0,90)$ & 2600 [17] & $\begin{array}{c}2892 \\
(+11 \%)\end{array}$ & $\begin{array}{c}2619 \\
(+0.7 \%)\end{array}$ & $\begin{array}{c}0.08 \text { [17], } \\
0.14[18]\end{array}$ & 0 & 0.40 \\
\hline$(0,138.5,26.7)$ & 2741 [19] & $\begin{array}{l}2808 \\
(+2 \%)\end{array}$ & $\begin{array}{c}2760 \\
(+0.9 \%)\end{array}$ & $\begin{array}{l}0.34[20], \\
0.44[19]\end{array}$ & 0.1 & 0.38 \\
\hline
\end{tabular}

Also shown in Table I is the electromechanical coupling coefficient calculated from the change in propagation velocity between a free surface and a surface with the boundary condition $V=0$, where $V$ is the electrostatic potential. The electromechanical coupling coefficient is calculated from the change in velocity through [21]

$$
K^{2}=\frac{\Delta v}{2 v_{0}}
$$

where $\Delta v$ is the velocity difference between free and shorted surfaces. As in the case of the propagation velocity, the best agreement with measurements is obtained for the $3 \mathrm{D}$ calculation. The error in the 2D simulation is quite significant for the $(0,138.5,26.7)$ Euler angle commonly used in langasite SAW sensors. In the following we use the 3D simulation only. 
We now consider surface acoustic wave propagation in langasite along the $(0,138.5,26.7)$ direction with a conducting overlayer. Simulations were performed for both $\mathrm{ZnO}$ and $\mathrm{TiO}_{2}$ overlayers. The layer thickness was $100 \mathrm{~nm}$ in both cases. The overlayer materials were assumed to be isotropic with material parameters shown in Table II.

TABLE II. MECHANICAL AND ELECTRICAL PARAMETERS FOR SENSING LAYERS USED IN SiMULATIONS.

\begin{tabular}{|c|c|c|c|c|}
\hline & $\varepsilon_{r}$ & $\rho\left[\mathrm{kg} / \mathrm{m}^{3}\right]$ & $v$ & $E[\mathrm{~Pa}]$ \\
\hline $\mathrm{TiO}_{2}$ & 85 & 4320 & 0.28 & $283 \times 10^{9}$ \\
\hline $\mathrm{ZnO}$ & 8.3 & 5676 & 0.33 & $210 \times 10^{9}$ \\
\hline
\end{tabular}

For both materials a surface wave bound to the surface was found (Figure 2). Figure 3 shows the predicted surface acoustic wave velocity for these two cases as a function of the layer conductivity. 

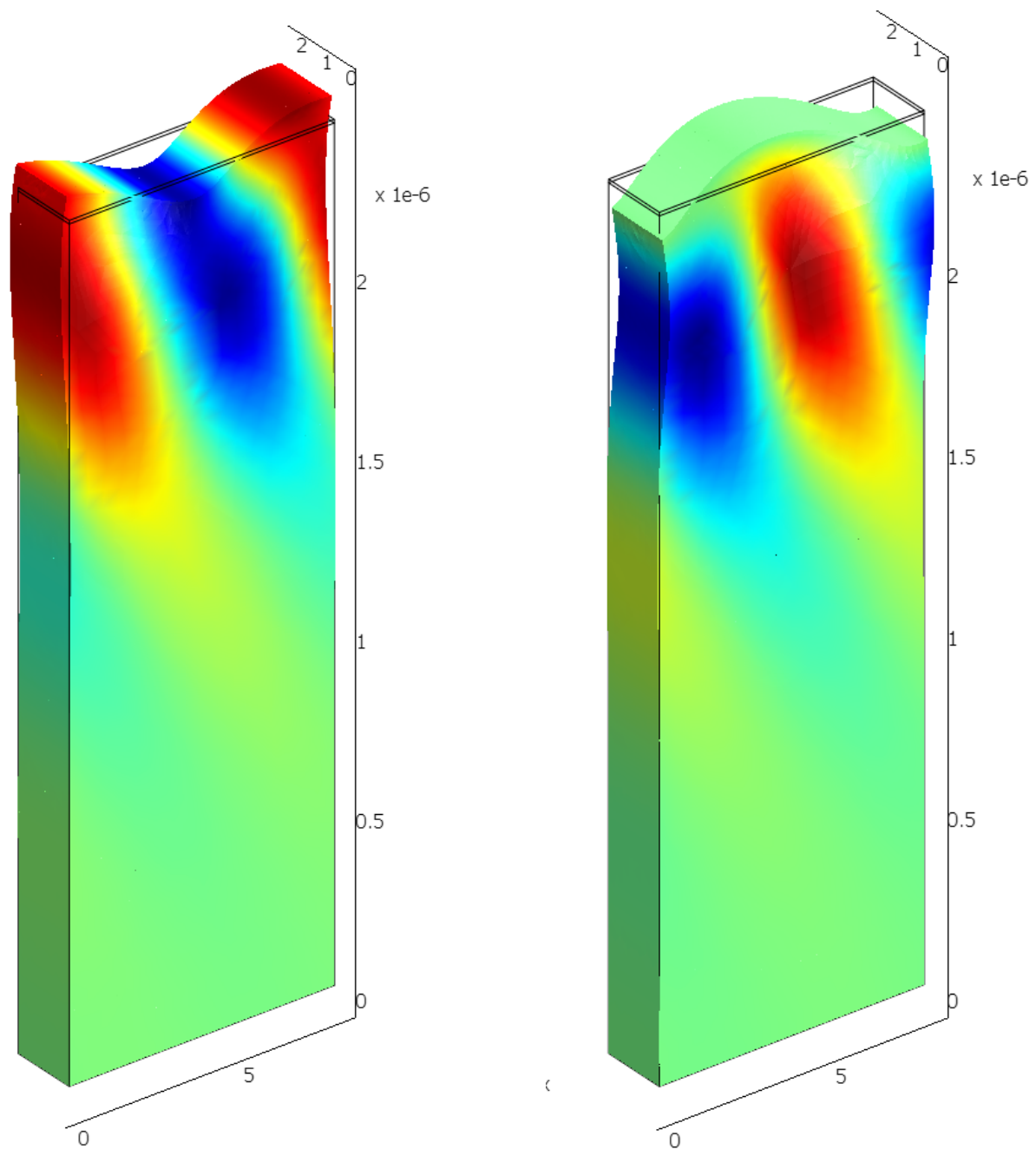

Figure 2. Simulated eigenmodes for $100 \mathrm{~nm} \mathrm{ZnO}$ on langasite (left, $348.5 \mathrm{MHz}$ ) insulating surface layer and (right, $347.9 \mathrm{MHz}$ ) highly conductive surface layer. Color represents electric potential and the distorted shape is shown. 


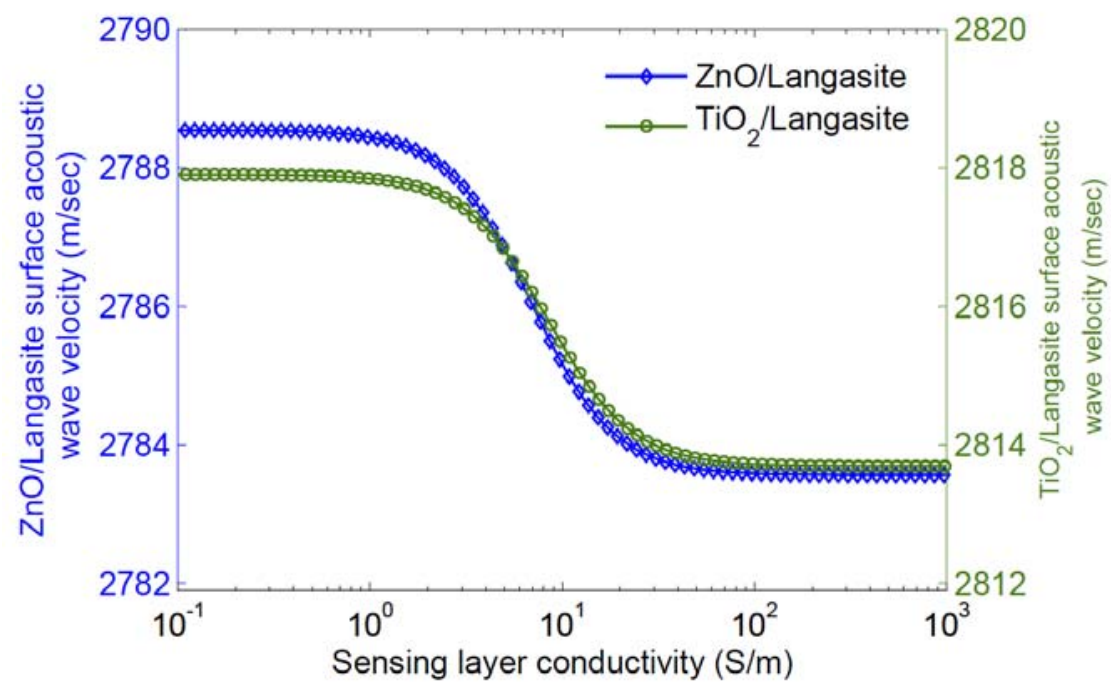

Figure 3. Dependence of velocity on film conductivity.

When the sensing layer conductivity is large the velocities are the same as the sensing layer is imposing a shorted-surface boundary condition. When the conductivity is low the surface charges cause piezoelectric stiffening, raising the velocity. This effect is more pronounced for a lower dielectric constant layer because for equal charges the electrostatic forces are reduced when the dielectric permittivity is increased. As a result a modestly greater change in velocity is predicted for $\mathrm{ZnO}$. The effect of the different mechanical stiffness of the two sensing layers is negligible.

The eigenmode calculations also permit calculation of the attenuation due to resistive losses in the sensing layer. ${ }^{\dagger}$ For a $100 \mathrm{~nm} \mathrm{ZnO}$ sensing layer, the peak loss is about $6 \mathrm{~dB} / \mathrm{mm}$ path length. This loss is substantially lower than would be obtained with a substrate with higher electromechanical coupling, and it acceptable for path lengths of a few mm as used in [8].

\section{Model Verification and Input Admittance}

In this section we report on measurements of the input admittance of interdigitated transducers in reflective delay-line devices on bare langasite substrate. Analysis of these measurements yields

† These calculations were performed using COMSOL 4.2, which yields the complex eigenfrequency. 
values for the propagation velocity and electromechanical coupling coefficent that can be compared with the three-dimensional simulations on bare langasite substrate discussed above. In addition, the input admittance is important when designing matching networks and/ or antennas.

Surface acoustic wave reflective delay-line devices were fabricated on langasite substrates oriented for propagation in the $(0,138.5,26.7)$ direction. These devices were later used as sensors for studies of wired and wireless sensing which are described elsewhere [4,5,9,8]. For now we focus on the characteristics of the emitting interdigitated transducer on bare langasite substrate.

The transducers consisted of e-beam deposited electrodes consisting of a $10 \mathrm{~nm}$ Ti adhesion layer followed by $100 \mathrm{~nm}$ Pt patterned by liftoff. The period of all transducers was $8 \mu \mathrm{m}$ with equal line and space dimensions. Four designs with different apertures and numbers of finger pairs were available as shown in Table III.

TABLE III. IDT PARAMETERS.

\begin{tabular}{|c|c|c|c|c|c|}
\hline Device ID & $N$ (pairs) & $L / \lambda$ & $k$ & $k^{2}$ & $v_{e}(\mathrm{~m} / \mathrm{sec})$ \\
\hline A1 & 20 & 100 & 0.059 & 0.0035 & 2612 \\
\hline A2 & 40 & 100 & 0.054 & 0.0029 & 2602 \\
\hline A3 & 50 & 100 & 0.055 & 0.0030 & 2618 \\
\hline B & 50 & 50 & 0.059 & 0.0035 & 2612 \\
\hline
\end{tabular}

The input admittance was measured at room temperature using a calibrated microwave probe (Micromanipulator 44-GP) and a Rohde and Schwartz ZVB4 network analyzer. Calibration was performed using a short, a known resistance, and an open at the probe tips. A total of 42 devices of four different types from the same wafer were measured.

There was significant variation in the measurements between individual devices of the same geometry. Figure 4 shows measurements of the conductance (real part of the admittance) as a function of frequency for all measured devices of type B. In general all devices showed a 
conductance peak at the same frequency and near $343 \mathrm{MHz}$, the expected resonant frequency. Most devices had clearly visible fine structure characteristic of reflections from nearby IDTs. In all cases the conductance peaks were superimposed on a near-constant background, indicating the presence of an additional loss mechanism. This excess conductance was nearly the same value for all devices with the exception of a few devices with higher conductance. We attribute this additional loss to various contributions to series resistance that will be discussed later.

Examination using the probe station microscope showed no obvious causes for inter-device variation, such as shorted or partially open fingers. However it is quite possible that defects of this type could be small and not easily seen. Other possible sources for variation include imperfect lift-off patterning and defects in the langasite substrate.

For further analysis we selected one device of each type that exhibited a narrow and high conductance peak and a typical value of the excess loss conductance. The data from the selected device is shown with a bold line in Figure 4. 


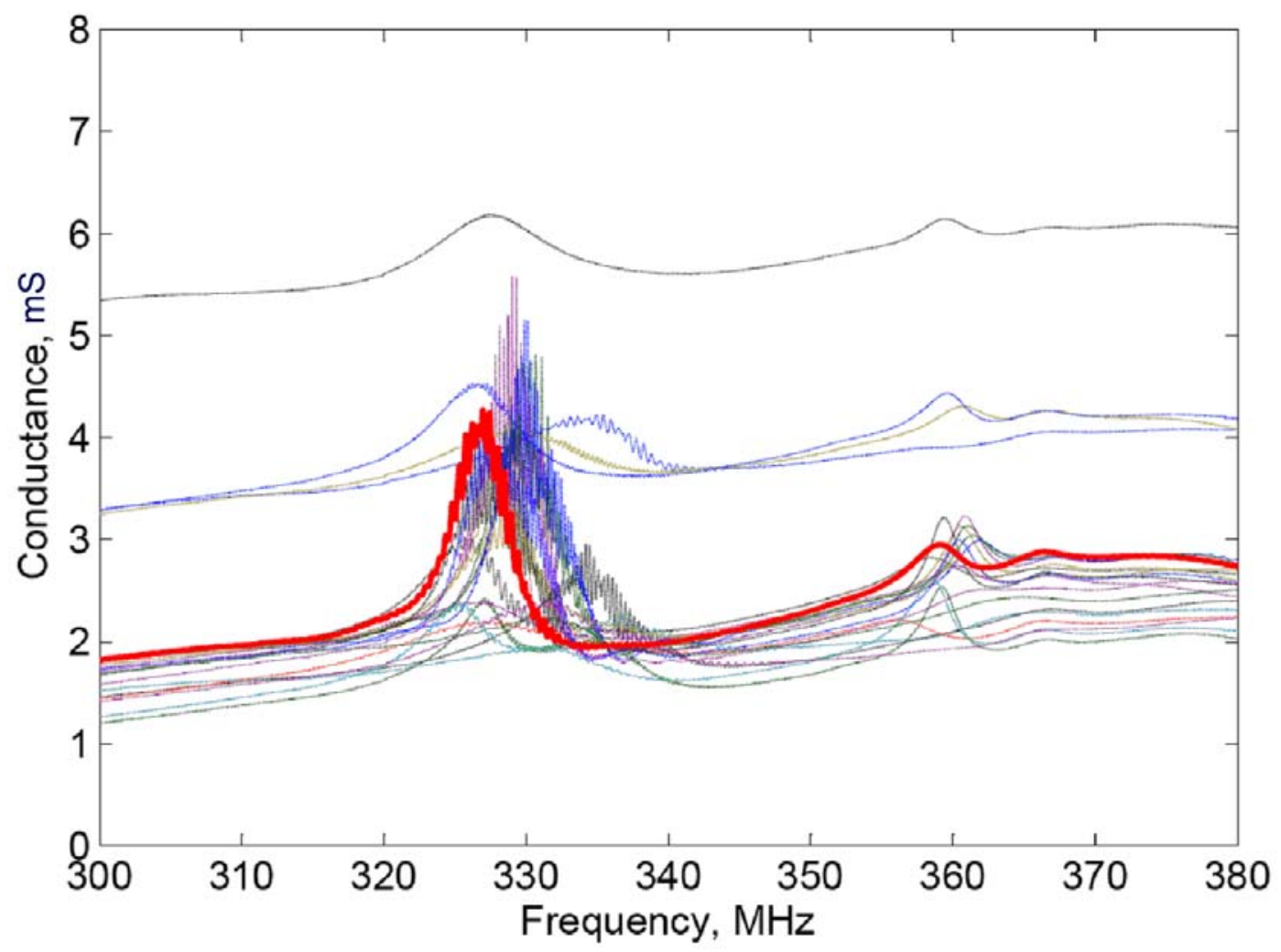

Figure 4. Measurements of the real part of the admittance for the $B$ series of transducers. The bold line shows the device selected for detailed analysis.

An additional small conductance peak near $360 \mathrm{MHz}$ is apparent in Figure 4 and also in data for the other IDTs. This is probably associated with an SH-bulk acoustic wave, as such waves have been previously reported in langasite. On other orientations this wave is efficiently excited [22] while here it is fairly weak. In 3D simulations this wave appears near $366 \mathrm{MHz}$, and exhibits displacement in the transverse direction (Figure 5). 


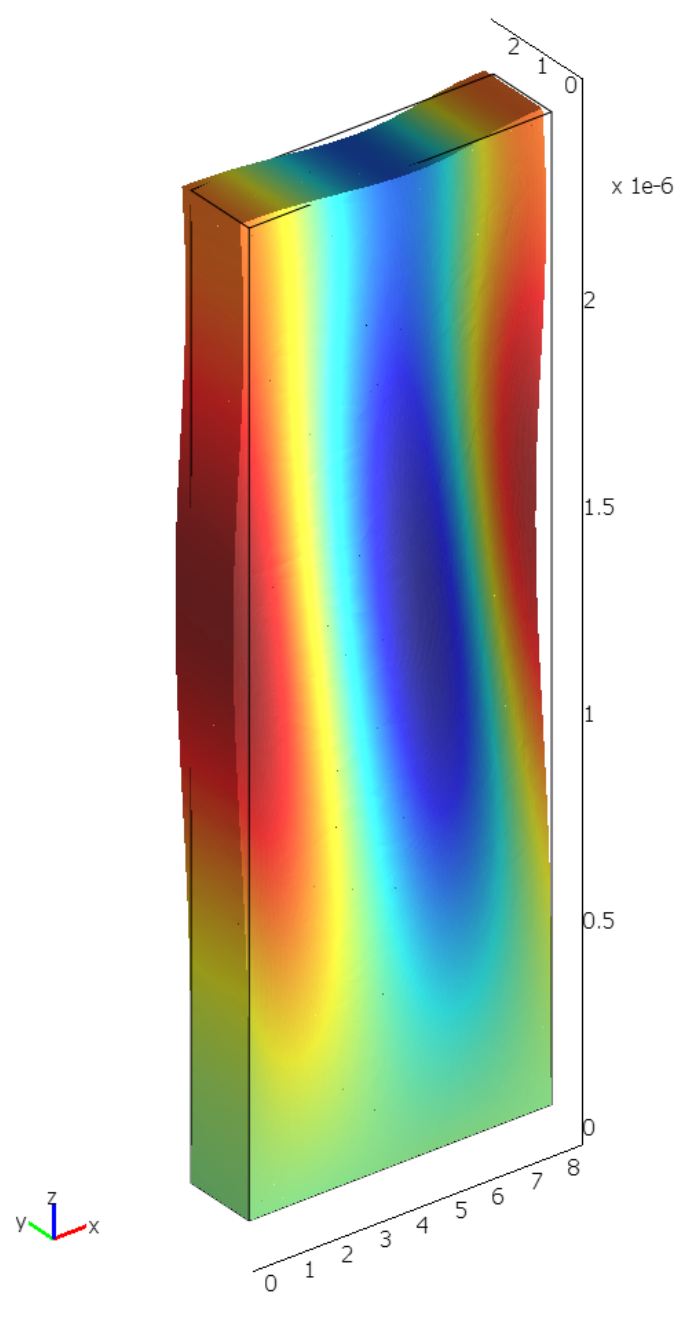

Figure 5. Eigenmode at $366 \mathrm{MHz}$ with displacement in the transverse direction.

The conductance peaks for the representative devices were fit using the theoretical expression for the real part of the admittance obtained by cascading $N$ pairs of fingers using the $P$-matrix formulation [23] or equivalently using the approximate expression [24]

$$
G(\omega)=\frac{8 \omega_{0} C_{s} k^{2} N^{2}}{2 \pi} \cdot\left(\frac{\sin (x)}{x}\right)^{2}
$$

where $x=N \pi\left(\omega-\omega_{0}\right) / \omega_{0}$ with $\omega$ the angular frequency, $\omega_{0}$ is the center frequency of the IDT, $N$ the number of finger pairs, and $C_{s}$ the finger capacitance per unit length. 
Apart from the structural parameters, the expression (5) has three adjustable parameters: the center frequency $\omega_{0}$, the electromechanical coupling coefficient $k^{2}$, and the finger capacitance per unit length $C_{s}$. The transducer resonant frequency is $325 \mathrm{MHz}$ and is consistent with the surface wave velocities in Table I and the finger spacing $d$. The capacitance $C_{s}=2.2 \times 10^{-12} \mathrm{~F} / \mathrm{cm}$ based on electrostatic simulations for the finger geometry, and this value is consistent with $1 \mathrm{MHz}$ capacitance measurements on the transducers. This leaves only the electromechanical coupling coefficient to be determined from a fit of the measured conductance data. The data has been fit by manually adjusting the value of the electromechanical coupling coefficient to obtain a good match to the envelope of the measured conductance peak.
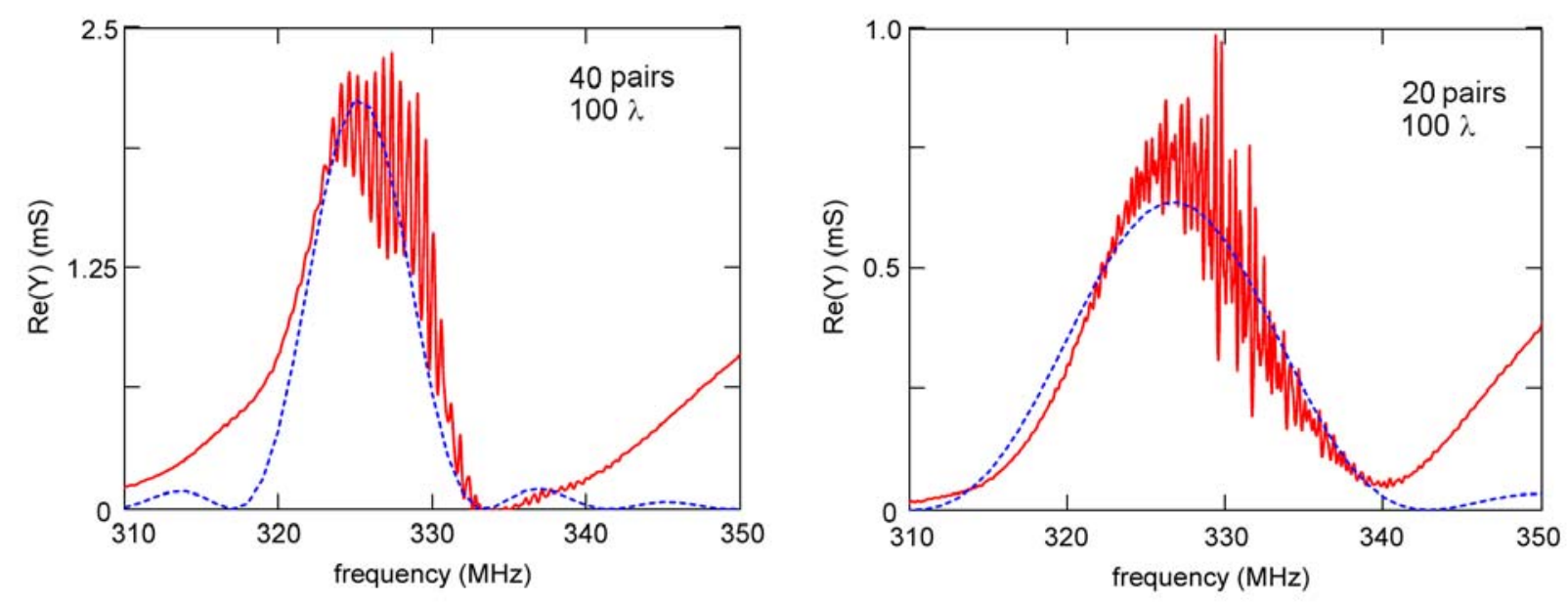

Figure 6. Fits to the real part of the measured admittance for transducers A1 and A2 (solid lines: measured data; the dashed line is the fit). The excess loss conductance has been subtracted from the data.

The extracted values for the electromechanical coupling coefficient and the surface wave velocity are shown in Table III. These results are in reasonable agreement with previous reports and the simulations presented above.

Because of the relatively small electromechanical coupling coefficient, the input admittance of langasite transducers is mostly capacitive. A theoretical expression for the capacitance can also be obtained from the $P$-matrix formulation. The measured capacitance along with the fit are 
shown in Figure 7 for the transducer designs A1 and A2 described in Table III. As $v_{s}$ and $K^{2}$ were extracted from the conductance measurements there are no adjustable parameters. Again, reasonable agreement is obtained, with some of the discrepancy attributable to the finger capacitance $C_{s}$.
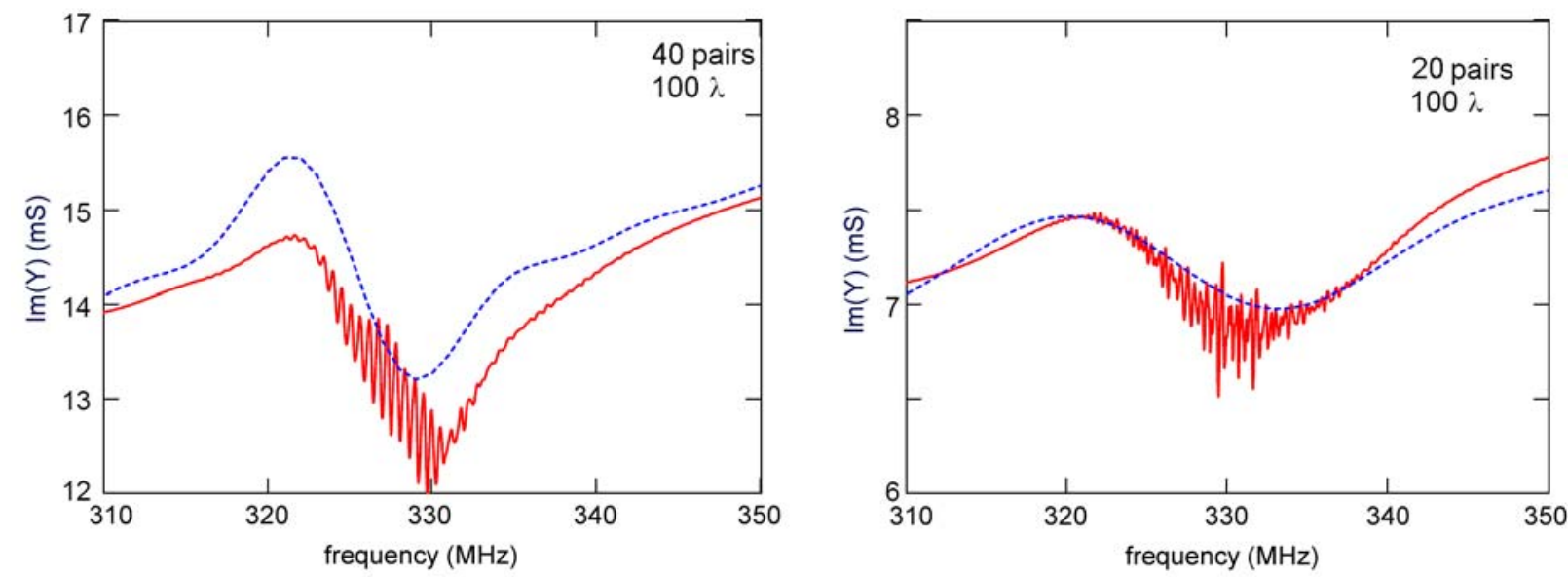

Figure 7. Measured capacitance for transducers A1 and A2 (solid line) along with fit (dashed line).

Finally, we turn to the excess conductance apparent in Figure 4. We attribute the excess conductance is to various distributed and lumped contributions to series resistance: (1) distributed loss in the fingers; (2) distributed loss in the bus-bars; and (3) additional lumped resistance associated with interconnect and probes. The last two contributions can be minimized by improved designs for the bus-bars and larger contact areas. However the finger resistance is likely to be a problem in most langasite SAW sensors. In the appendix we summarize an approximate calculation of the losses due to finger resistance. The excess conductance due to resistive losses in the fingers is calculated in the Appendix and the results are shown in Table IV. The finger loss accounts for most of the excess loss for IDTs with long fingers and for some of the excess loss in the IDT with short fingers. The remaining loss is attributed to loss in the busbars and contacts. 
TABLE IV. COMPARISON OF CONDUCTANCE DUE TO THE FINGER SERIES RESISTANCE AND THE MEASURED EXCESS CONDUCTANCE.

\begin{tabular}{|c|c|c|c|}
\hline aperture $(\lambda)$ & finger pairs & $\mathrm{G}$ (calculated, $\mathrm{mS}$ ) & $\mathrm{G}$ (measured, $\mathrm{mS}$ ) \\
\hline 50 & 50 & 0.5 & 2.0 \\
\hline 100 & 50 & 3.8 & 5.9 \\
\hline 100 & 40 & 3.0 & 4.5 \\
\hline 100 & 20 & 1.5 & 2.4 \\
\hline
\end{tabular}

Langasite has a permittivity comparable to lithium niobate but interconnect resistances are likely to be much greater. Platinum is a commonly used metallization which has a bulk electrical conductivity roughly a factor of four higher than aluminum and is likely to be used in thinner layers because of its cost. Other proposed high-temperature metallizations include alloys [25] and may well exhibit additional scattering and thus lower conductivity. High capacitance per unit length along with higher resistance per unit length can lead to unacceptable distributed losses. These losses will be more apparent for SAW devices operated at frequencies of hundreds of $\mathrm{MHz}$ as used here although they may be minimal at low frequencies [26]. These considerations suggest that the width of IDTs in langasite SAW devices should be limited and that the sheet resistance of the metallization is an important parameter.

\section{Conclusions}

We have performed finite element calculations relating the surface wave velocity change to the conductivity of a sensing layer. For the commonly used (0, 138.5, 26.7) Euler angle direction, three-dimensional calculations are necessary because of the non-negligible transverse displacements. Calculations using the material parameters for both $\mathrm{ZnO}$ and $\mathrm{TiO}_{2}$ have been made. The calculations include both the mechanical and electrical influence of the sensing layer. Velocity changes are large enough to be readily measured. Because of the relatively small electromechanical coupling coefficient of langasite, the wave attenuation associated with the resistive losses is not very significant unless the path length is quite long. The simulation results predicting velocity change as a function of sensing layer conductivity can be used to guide conductivity based langasite surface acoustic wave sensor design. 
Input admittance measurements on several different interdigitated transducers were analyzed to extract an experimental value for the electromechanical coupling coefficient which is in good agreement with the three-dimensional simulations on bare langasite substrate. Admittance measurements also show excess losses which are attributed to resistive losses in the fingers and other parts of the interconnect. These losses are likely to be significant in langasite SAW devices because commonly used metallizations such as platinum have relatively high resistivity. Smaller IDT width and larger interconnect contact area is suggested to reduce the resistive losses in high frequency langasite IDT design.

\section{Acknowledgements}

We acknowledge the contribution of Limin Cao who performed wafer fabrication. This project was funded by the Department of Energy, National Energy Technology Laboratory, an agency of the United States of Government, through a support contract with URS Energy \& Construction, Inc. Neither the United States Government nor any agency thereof, nor any of their employees, nor URS Energy \& Construction, Inc., nor any of their employees, makes any warranty, expressed or implied, or assumes any legal liability or responsibility for the accuracy, completeness, or usefulness of any information, apparatus, product, or process disclosed, or represents that its use would not infringe privately owned rights. Reference herein to any specific commercial product, process, or service by the trade name, trademark, manufacturer, or otherwise, does not necessarily constitute or imply its endorsement, recommendation, or favoring by the United States Government or any agency thereof. The views and opinions of authors expressed herein do not necessarily state or reflect those of the United States Government or any agency thereof.

\section{Appendix}


In this section we estimate the loss due to finger resistance in a SAW device away from the IDT resonant frequency. In the case of substrate materials with small electromechanical coupling such as langasite, and especially away from resonance, the finger impedance is mostly capacitive. We approximately model the fingers as a capacitor (that is, the radiation resistance is neglected).

Figure 8 shows the equivalent circuit for one finger subject to this approximation. The capacitance $C$ is the capacitance per unit length between one finger and the neighboring two fingers and $R$ is the resistance per unit length of the finger. The finger is driven by a voltage source $V$ and the neighboring two fingers are grounded at the opposite ends. The top string of resistors represents the driven finger with a voltage $v_{2}(x)$ varying along the finger; $v_{1}(x)$ is the corresponding voltage for the two neighboring grounded fingers.

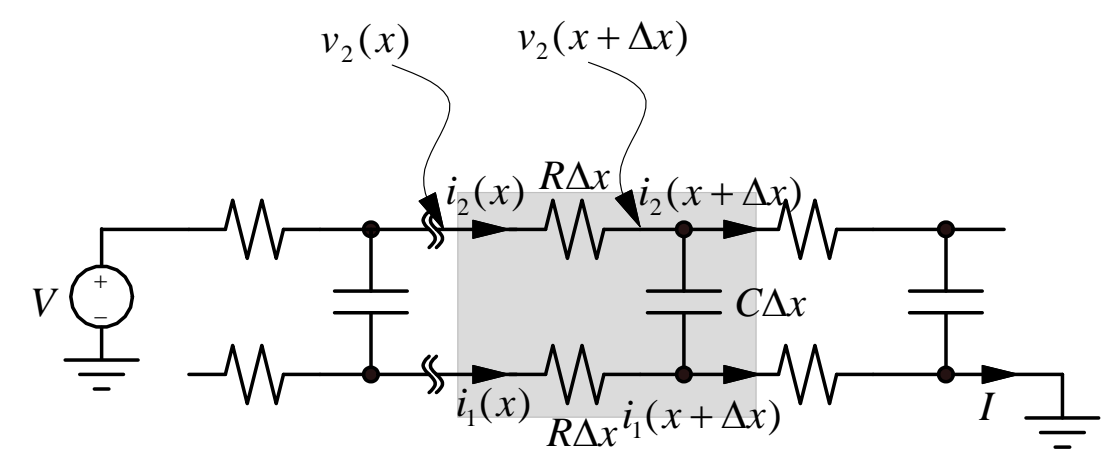

Figure 8. Equivalent circuit representing one finger. The shaded area shows one section of length $\Delta x$.

Writing Kirchoff's current and voltage laws at a node at position $x$ and combining equations yields the differential equation

$$
\frac{\partial^{2}\left(v_{2}-v_{1}\right)}{\partial x^{2}}=2 j \omega C R\left(v_{2}-v_{1}\right)
$$

The general solution to the differential equation is of the form

$$
v_{2}-v_{1}=A e^{(1+j) a x}+B e^{-(1+j) a x}
$$


where

$$
a=\sqrt{\omega R C}
$$

The same current $I$ enters at the left from the voltage source and leaves at the right to ground. As a result we have the boundary conditions

$$
\begin{aligned}
& \left.\frac{\partial\left(v_{2}-v_{1}\right)}{\partial x}\right|_{x=0}=-R I \\
& \left.\frac{\partial\left(v_{2}-v_{1}\right)}{\partial x}\right|_{x=L}=R I
\end{aligned}
$$

where $L$ is the total finger width. This gives the solution for $v_{2}-v_{1}$

$$
v_{2}-v_{1}=\frac{R I}{2 a}(1+j)\left[\frac{e^{(1+j) a x}}{e^{(1+j) a L}-1}+\frac{e^{-(1+j) a x}}{1-e^{-(1+j) L}}\right]
$$

Physically $v_{2}-v_{1}$ is the voltage that appears between the fingers so we would like this to remain large and constant for most effective excitation of the surface wave. The quantity $a$ is a reciprocal decay length. When $a<<1 / L$ the potential difference is uniform along the fingers. When $a$ approaches $1 / L$ then excitation is less effective.

This solution can be used to determine the finger admittance $Y=I / v_{2}(0)$. This calculation is straightforward but involved as $v_{2}(0)$ is found by integration

$$
v_{2}(0)=R \int_{0}^{L} i_{2} d x+\left.\left(v_{2}-v_{1}\right)\right|_{x=L}
$$

where the current $i_{2}(\mathrm{x})$ is 


$$
i_{2}(x)=I-j \omega C \int_{0}^{x}\left(v_{2}-v_{1}\right) d x
$$

The results were presented in Table IV for IDTs with $100 \mathrm{~nm}$ Pt metallization where we have used the measured platinum sheet resistance from metal lines of known width and length to determine $R$.

\section{References}

1, S.-Q. Wang, J. Harada and S. Uda, “A Wireless Surface Acoustic Wave Temperature Sensor Using Langasite as Substrate Material for High-Temperature Applications,” Jpn. J. Appl. Phys. Vol. 42, pp. 6124-6127 Part 1, No. 9B, September 2003.

2. J.A. Thiele, and M. Pereira da Cunha, "High Temperature SAW Gas Sensor on Langasite," Proceedings of IEEE Sensors 2003, pp. 769-772.

3. A. Canabal, P.M. Davulis, E. Dudzik, and M. Pereira da Cunha, ”CDMA and FSCW Surface Acoustic Wave Temperature Sensors for Wireless Operation at High Temperatures," IEEE International Ultrasonics Symposium Proceedings 2009 pp. 807-810.

4. T.L. Chin, D. P. Zheng, W. Greve, L. Cao, and I. J. Oppenheim, “Flexible instrumentation for wireless SAW,” in 2010 Proc. IEEE International Ultrasonic Symposium, pp. 261-264.

5. P. Zheng, D.W. Greve, I.J. Oppenheim, and V. Malone, "Langasite SAW temperature and oxygen multi-sensor," Proc. 2011 IEEE Frequency Control Conference, digital object identifier 10.1109/FCS.2011.5977811, pp. not available yet.

6. Tortissier, L. Blanca, A. Tetelina,, J.-L. Lachauda, M. Benoitb, V. Conédéra, C. Dejousa, D. Rebièrea, "Langasite based surface acoustic wave sensors for high temperature chemical detection in harsh environment: Design of the transducers and packaging,” Sensors and Actuators B, Vol. 156, pp. 510- 516, 2011.

7. J. A. Thiele and M. Pereira da Cunha, "High Temperature LGS SAW devices with Pt/WO3 and Pd Sensing Films,” IEEE Ultrasonics Symposium 2003, pp.1750-1753. 
8. P. Zheng, T.-L. Chin, D. Greve, I. Oppenheim, V. Malone, and L. Cao, "High Temperature Langasite SAW Oxygen Sensor," IEEE Trans. Ultrasonics, Ferrroelectrics, and Frequency Control, vol. 58, pp. 1538-40, 2011.

9. Zheng, P., Chin, T.-L., Greve, D. W., Oppenheim, I. J., Ashok, T., Malone, V., Miller, J., Cao, L. (2010). “Langasite SAW Device with Gas-Sensitive Layer”, IEEE Ultrasonics Symposium 2010 Proceedings.

10. P. Zheng, D.W. Greve, I.J. Oppenheim, T.-L. Chin, and V. Malone, "Langasite Surface Acoustic Wave Sensors: Fabrication and Testing,” IEEE Transactions on Ultrasonics, Ferroelectrics, and Frequency Control, vol. 59, issue 2, pp. 295-303, 2012.

11. R. Lec, J.F. Vetelino, R.S. Falconer, and Z. Xu, "Macroscopic Theory of surface acoustic wave gas microsensors,” 1988 IEEE Ultrasonics Symposium, pp. 585-589.

12. Michele Penza, Eva Milella, and Vladimir I. Anisimkin, "Gas Sensing Properties of Langmuir-Blodgett Polypyrrole Film Investigated by Surface Acoustic Waves,” IEEE

Transactions on Ultrasonics, Ferroelectrics, and Frequency Control, vol. 45, no. 5, september pp. 1125-1132, 1998.

13. For example, D.M. Smyth, Titanium dioxide. in The Defect Chemistry of Metal Oxides;

Oxford University Press: New York, NY, USA, 2000; pp. 217-238.

14. D. S. Ballantine, Acoustic Wave Sensors - Theory, Design, and Physico-Chemical Applications: Elsevier, 1997.

15. J.A. Kosinski, R. A. Pastore, E. Bigler, M. Pereira da Cunha, D.C. Malocha, and J. Detaint, "A Review of Langasite Material Constrants from BAW and SAW Data: Toward an Improved Data Set," 2001 IEEE International Frequency Control Symposium Proceedings, pp. 278-286 (2001).

16. E. Chilla, C. M. Flannery, and H.-J. Fröhlich, "Elastic properties of langasite-type crystals determined by bulk and surface acoustic waves," JOURNAL OF APPLIED PHYSICS, vol. 90, pp. 6084-6091, 2001.

17. M. Adachi, T. Karaki, and W. Miyamoto, "Surface Acoustic Wave Properties of La3Ga5SiO14 (langasite) Single Crystals," Jpn. J. Appl. Phys., vol. 38, pp. 3283-3287, 1999. 
18. K. Inoue and K. Sato, "Propagation Characteristics of Surface Acoustic Waves on Langasite," Jpn. J. Appl. Phys., vol. 37, pp. 2909-2913, 1998.

19. J. Bardong, M. Schulz, M. Schmitt, I. Shrena, D. Eisele, E. Mayer, L. M. Reindl, and H. Fritze, "Precise Measurements of BAW and SAW Properties of Langasite in the Temperature Range from $25^{\circ} \mathrm{C}$ to $1000{ }^{\circ} \mathrm{C}, " 2008$ IEEE International Frequency Control Symposium, pp. 326-331, 2008.

20. N. Naumenko, "Optimal Cuts of Langasite, La3Ga5SiO14 for SAW Devices," IEEE Transactions on Ultrasonics, Ferroelectrics, and Frequency Control, vol. 48, 2001.

21. K.A. Ingebrigtsen, "Analysis of Interdigital Transducers," 1972 IEEE Ultrasonics Symposium, pp. 403-407.

22. T. D. Kenny, T. B. Pollard, E. J. Berkenpas and M. Pereira da Cunha, "FEM/BEM impedance and power analysis for measured LGS SH-SAW devices”, Proceedings of the IEEE International Conference on Ultrasonics, Ferroelectrics and Frequency Control, pp. 1371-1374, 2004.

23. G. Tobolka, “Mixed Matrix Representation of SAW Transducers,” IEEE Trans. Sonics and Ultrasonics vol. SU-26, no. 6, pp. 426-427, 1979.

24. W.R. Smith, H.M. Gerard, J.H. Collins, T.M. Reeder, and H.J. Shaw, "Analysis of Interdigital Surface Wave Transducers by use of an Equivalent Circuit Model," IEEE Transactions on Microwave Theory and Techniques, vol. 17, pp. 856-864, Nov. 1969.

25. D.J. Frankel, G.P. Bernhardt, B.T. Sturtevant, T. Moonlight, M. Pereira da Cunha, R.J. Lad, "Stable Electrodes and Ultrathin Passivation Coatings for High Temperature Sensors in Harsh Environments." Proc. 2008 IEEE Sensors, pp. 82-85.

26. D. Puccio, D.C. Malocha, N. Saldanha, and M. Pereira da Cunha, "SAW Parameters on Ycut LangasiteStructured Materials,” IEEE Transactions on Ultrasonics, Ferroelectrics, and Frequency Control, vol. 54, no. 9, pp. 1873-1881, September, 2007. 